\title{
EXISTENCE AND WEAK-TYPE INEQUALITIES FOR CAUCHY INTEGRALS OF GENERAL MEASURES ON RECTIFIABLE CURVES AND SETS
}

\author{
PERTTI MATTILA AND MARK S. MELNIKOV
}

(Communicated by J. Marshall Ash)

ABstract. If $\mu$ is a finite complex Borel measure and $\Gamma$ a Lipschitz graph in the complex plane, then for $\lambda>0$

$$
\left|\left\{z \in \Gamma: \sup _{\varepsilon>0}\left|\int_{|\zeta-z| \geq \varepsilon}(\zeta-z)^{-1} d \mu \zeta\right|>\lambda\right\}\right| \leq c(\Gamma) \lambda^{-1}\|\mu\|_{1} .
$$

It follows that for any finite Borel measure $\mu$ and any rectifiable curve $\Gamma$ the finite principal value

$$
\lim _{\varepsilon \downarrow 0} \int_{|\zeta-z| \geq \varepsilon}(\zeta-z)^{-1} d \mu \zeta
$$

exists for almost all (with respect to length) $z \in \Gamma$.

\section{INTRODUCTION}

For any finite complex Borel measure $\mu$ on the complex plane $\mathbb{C}$ the Cauchy transform

$$
\hat{\mu}(z)=\int(\zeta-z)^{-1} d \mu \zeta
$$

exists for almost all $z \in \mathbb{C}$ with respect to area. This is a rather immediate consequence of the fact that the kernel $z^{-1}$ is locally integrable with respect to the Lebesgue measure. In this paper we prove that much more is true provided we interpret the Cauchy integral as a principal value. Namely, for any rectifiable curve $\Gamma$, the limit

$$
C_{\mu}(z)=\lim _{\varepsilon \downarrow 0} \int_{|\zeta-z| \geq \varepsilon}(\zeta-z)^{-1} d \mu \zeta
$$

exists and is finite for $\mathscr{H}^{1}$ almost all $z \in \Gamma$. Here $\mathscr{H}^{1}$ is the one-dimensional Hausdorff (i.e., length) measure on $\mathbb{C}$. This result follows from the following weak-type inequality, which we prove in $\S 2$. Define, for $\varepsilon>0, z \in \mathbb{C}$,

$$
\begin{aligned}
C_{\mu, \varepsilon}(z) & =\int_{\mathbb{C} \backslash B(z, \varepsilon)}(\zeta-z)^{-1} d \mu \zeta, \\
C_{\mu}^{*}(z) & =\sup _{\varepsilon>0}\left|C_{\mu, \varepsilon}(z)\right|,
\end{aligned}
$$

Received by the editors July 9, 1991 and, in revised form, February 5, 1992 and April 24, 1992. 1991 Mathematics Subject Classification. Primary 30E20; Secondary 28A75, 42B20. 
where $B(z, \varepsilon)=\{\zeta:|\zeta-z| \leq \varepsilon\}$. Then, for any Lipschitz graph $\Gamma$ and $\lambda>0$,

$$
\mathscr{H}^{1}\left\{z \in \Gamma: C_{\mu}^{*}(z)>\lambda\right\} \leq c \lambda^{-1}\|\mu\|_{1} .
$$

Here $c$ is a finite constant depending only on the Lipschitz constant of $\Gamma$ and $\|\mu\|_{1}$ stands for the variation norm of $\mu$; that is, $\|\mu\|_{1}=|\mu|(\mathbb{C})$, with $|\mu|$ denoting the total variation measure of $\mu$.

If $\mu$ is supported by the same curve $\Gamma$, the above results follow from the well-known work of Calderón and others; see [C, CM, D2]. In particular, the Cauchy principal values

$$
C_{\Gamma} f(z)=\lim _{\varepsilon \downarrow 0} \int_{\Gamma \backslash B(z, \varepsilon)}(\zeta-z)^{-1} f(\zeta) d \mathscr{H}^{1} \zeta
$$

exist for $\mathscr{H}^{1}$ almost all $z \in \Gamma$ for $f \in L^{1}(\Gamma)$ if $\Gamma$ is a rectifiable curve. An immediate consequence of our results is that the same holds for any rectifiable $\mathscr{H}^{1}$ measurable set $E$. By saying that $E$ is rectifiable we mean that $\mathscr{H}^{1}(E)<$ $\infty$ and that there are rectifiable curves $\Gamma_{1}, \Gamma_{2}, \ldots$ such that $\mathscr{l}^{1}\left(E \backslash \bigcup_{i=1}^{\infty} \Gamma_{i}\right)=$ 0 . This class of sets was first extensively studied by Besicovitch who called them regular; see, for example, [F]. Although an exact converse is not known, it seems that, as for the existence of Cauchy principal values almost everywhere on $E$, the rectifiable sets $E$ may form the optimal class. As a partial result it was shown in [M] that, if $E$ is $\mathscr{H}^{1}$ measurable, $\mathscr{H}^{1}(E)<\infty$,

$$
\liminf _{r \downarrow 0} r^{-1} \mathscr{H}^{1}(E \cap B(z, r))>0 \text { for } \mathscr{H}^{1} \text { almost all } z \in E,
$$

and

$$
\exists \lim _{\varepsilon \downarrow 0} \int_{E \backslash B(z, \varepsilon)}(\zeta-z)^{-1} d \mathscr{H}^{1} \zeta \in \mathbb{C} \text { for } \mathscr{H}^{1} \text { almost all } z \in E,
$$

then $E$ is rectifiable. Since (2) holds for any rectifiable set, we obtain the following characterization of rectifiability improving the one given in [M, 4.22]:

An $\mathscr{H}^{1}$ measurable subset $E$ of $\mathbb{C}$ with $\mathscr{H}^{1}(E)<\infty$ is rectifiable if and only if (2) and (3) hold.

It is not known whether (2) could be deleted from this characterization.

In [D1] David studied the boundedness of $C_{\mu}$ from $L^{p}(\mu)$ into $L^{p}(\Gamma)$ for $1<p<\infty$ with some conditions on the measure $\mu$ and curve $\Gamma$. In fact, he considered more general kernels. Later on, further generalizations, also to higher dimensions, were derived by David and Semmes; see [D2]. It seems probable that also the methods of our paper could be considerably extended.

The basic idea in the proof of (1) consists of projecting the measure $\mu$ to an $L^{1}$ function $g$ on the curve $\Gamma$, applying known results for the Cauchy integral of $g$, and estimating the difference of the Cauchy integrals of $g$ and $\mu$.

We would like to thank the referee for suggesting a simplification to our original proof.

\section{WEAK-TYPE INEQUALITIES ON LIPSCHITZ GRAPHS}

Let $\Gamma$ be the graph of a Lipschitz function $f: I \rightarrow \mathbb{R}$ where $I$ is an interval on the real line $\mathbb{R}$. Using the notation of the introduction, we now prove 
2.1. Theorem. Let $\mu$ be a finite complex measure on $\mathbb{C}$. Then, for any $\lambda>0$,

$$
\mathscr{H}^{1}\left\{z \in \Gamma: C_{\mu}^{*}(z)>\lambda\right\} \leq c \lambda^{-1}\|\mu\|_{1},
$$

where $c$ is a constant depending only on the Lipschitz constant of $f$.

Proof. Obviously we may assume $I=\mathbb{R}$. Fix $L, 1<L<\infty$, such that

$$
|f(x)-f(y)| \leq L|x-y| \text { for } x, y \in \mathbb{R} .
$$

$c_{1}, c_{2}, \ldots$ will denote positive and finite constants depending only on $L$. We can write $\mu=\mu_{1}+\mu_{2}$, where $\mu_{1}$ is the restriction of $\mu$ to $\Gamma$ and $\left|\mu_{2}\right|(\Gamma)=0$. Inequality (1) holds for $\mu_{1}$ by the well-known results; see, for example, [C, CM, D2, MT]. In fact, it is usually stated for $L^{1}$ functions on $\Gamma$, but it extends easily by approximation for general measures on $\Gamma$. Thus we may assume $|\mu|(\Gamma)=0$.

We shall use a Whitney-type decomposition of $\mathbb{C} \backslash \Gamma$. We may assume that $\mu$ is concentrated on $\{x+i y: y>f(x)\}$, and we shall decompose only this part.

Define for all integers $m$ and $j$

$$
\begin{aligned}
I_{m, j} & \left.=](j-1) 2^{-m}, j 2^{-m}\right], \\
R_{m, j} & =\left\{x+i y: x \in I_{m, j}, L 2^{1-m}+f(x) \leq y<L 2^{2-m}+f(x)\right\}, \\
R_{m} & =\bigcup_{j \in \mathbb{Z}} R_{m, j} .
\end{aligned}
$$

Denote

$$
\mu_{m, j}=\mu\left(R_{m, j}\right), \quad M_{m, j}=|\mu|\left(R_{m, j}\right), \quad \Gamma_{m, j}=\left\{x+i f(x): x \in I_{m, j}\right\} .
$$

For $z \in \Gamma$ we let $j_{m}(z)$ be the unique integer such that $z \in \Gamma_{m, j_{m}(z)}$. Note that

$$
\begin{array}{ll}
2^{-m} \leq|z-\zeta| \leq L 2^{2-m} & \text { for } z \in \Gamma_{m, j}, \quad \zeta \in R_{m, j} \\
|z-\zeta| \geq(|j-k|-1) 2^{-m} & \text { for } z \in \Gamma_{m, j}, \quad \zeta \in R_{m, k} \\
\left|\int_{R_{m, j}}(\zeta-z)^{-1} d \mu \zeta\right| \leq 2^{m} M_{m, j} & \text { for } z \in \Gamma .
\end{array}
$$

We define

$$
\begin{gathered}
a_{m, j}=\mu_{m, j} / \mathscr{H}^{1}\left(\Gamma_{m, j}\right), \\
g_{m}: \Gamma \rightarrow \mathbb{C}, \quad g_{m}=a_{m, j} \quad \text { on } \Gamma_{m, j}, \\
g=\sum_{m \in \mathbb{Z}} g_{m}, \quad h=\sum_{m \in \mathbb{Z}}\left|g_{m}\right|
\end{gathered}
$$

and observe that

$$
\|g\|_{1} \leq\|h\|_{1} \leq \sum_{j, m} M_{m, j}=\|\mu\|_{1} .
$$

Here \|\|$_{1}$ is the $L^{1}$ norm on $\Gamma$.

To prove the theorem we have to show that, for a given positive function $\varepsilon$ on $\Gamma$,

$$
\mathscr{H}^{1}\left\{z \in \Gamma:\left|C_{\mu, \varepsilon(z)}(z)\right|>\lambda\right\} \leq c_{1} \lambda^{-1}\|\mu\|_{1} .
$$


Clearly we may take $\varepsilon$ sufficiently regular-for example, piecewise constant-so that $z \mapsto C_{\mu, \varepsilon(z)}(z)$ is a Borel function. Let

$$
B_{z}=B(z, \varepsilon(z))
$$

and define

$$
\begin{aligned}
u_{m}(z) & =\sum_{j \in \mathbb{Z}}\left[\int_{R_{m}, j \backslash B_{z}}(\zeta-z)^{-1} d \mu \zeta-\int_{\Gamma_{m, j} \backslash B_{z}}(t-z)^{-1} a_{m, j} d t\right], \\
u(z) & =\sum\left\{u_{m}(z): L 2^{-m}>\varepsilon(z)\right\} \\
v(z) & =\sum\left\{u_{m}(z): L 2^{-m} \leq \varepsilon(z),\left|j-j_{m}(z)\right| \geq 2^{m+1} \varepsilon(z)\right\} \\
w(z) & =\sum\left\{u_{m}(z): L 2^{-m} \leq \varepsilon(z),\left|j-j_{m}(z)\right|<2^{m+1} \varepsilon(z)\right\}
\end{aligned}
$$

Then

$$
C_{\mu, \varepsilon(z)}(z)=u(z)+v(z)+w(z)+\int_{\Gamma \backslash B_{z}}(t-z)^{-1} g(t) d t
$$

We prove that

$$
\begin{gathered}
\|u\|_{1} \leq c_{2}\|\mu\|_{1}, \\
\|v\|_{1} \leq c_{3}\|\mu\|_{1}, \\
\mathscr{H}^{1}\{z \in \Gamma:|w(z)|>\lambda\} \leq c_{4} \lambda^{-1}\|\mu\|_{1} .
\end{gathered}
$$

Since by (6) and [C]

$$
\mathscr{H}^{1}\left\{z \in \Gamma:\left|\int_{\Gamma \backslash B_{z}}(t-z)^{-1} g(t) d t\right|>\lambda\right\} \leq c_{5} \lambda^{-1}\|\mu\|_{1},
$$

the theorem follows from these inequalities.

To verify (8) note that, if $\varepsilon(z)<L 2^{-m}$, then $R_{m, j} \cap B_{z}=\varnothing$ for all $j$ and $\Gamma_{m, j} \cap B_{z}=\varnothing$ for $\left|j-j_{m}(z)\right| \geq 2 L$. Hence,

$$
\begin{aligned}
\left|u_{m}(z)\right| \leq & \sum_{\left|j-j_{m}(z)\right| \geq 2 L}\left|\int_{R_{m, j}}(\zeta-z)^{-1} d \mu \zeta-\int_{\Gamma_{m, j}}(t-z)^{-1} a_{m, j} d t\right| \\
& +\sum_{\left|j-j_{m}(z)\right|<2 L}\left[\left|\int_{R_{m, j}}(\zeta-z)^{-1} d \mu \zeta\right|+\left|\int_{\Gamma_{m, j} \backslash B_{z}}(t-z)^{-1} a_{m, j} d t\right|\right] .
\end{aligned}
$$


Select $\zeta_{m, j} \in R_{m, j}$. Then for $\left|j-j_{m}(z)\right| \geq 2$ by (4)

$$
\begin{aligned}
\left|\int_{R_{m, j}}(\zeta-z)^{-1} d \mu \zeta-\int_{\Gamma_{m, j}}(t-z)^{-1} a_{m, j} d t\right| \\
\leq\left|\int_{R_{m, j}}(\zeta-z)^{-1} d \mu \zeta-\left(\zeta_{m, j}-z\right)^{-1} \mu_{m, j}\right| \\
\quad+\left|\left(\zeta_{m, j}-z\right)^{-1} \mu_{m, j}-\int_{\Gamma_{m, j}}(t-z)^{-1} a_{m, j} d t\right| \\
=\left|\int_{R_{m, j}} \frac{\zeta_{m, j}-\zeta}{(\zeta-z)\left(\zeta_{m, j}-z\right)} d \mu \zeta\right|+\left|\int_{\Gamma_{m, j}} \frac{\left(t-\zeta_{m, j}\right) a_{m, j}}{\left(\zeta_{m, j}-z\right)(t-z)} d t\right| \\
\leq c_{6}\left[\frac{4 L 2^{-m} M_{m, j}}{\left(\left(j-j_{m}(z)\right) 2^{-m}\right)^{2}}+\frac{8 L 2^{-m} \cdot 2^{1+m} M_{m, j} L 2^{-m}}{\left(\left(j-j_{m}(z)\right) 2^{-m}\right)^{2}}\right] \\
\leq c_{7} 2^{m} M_{m, j}\left(j-j_{m}(z)\right)^{-2} .
\end{aligned}
$$

Let $\widetilde{\Gamma}_{m, j}=\bigcup\left\{\Gamma_{m, i}:|j-i|<2 L\right\}$. Since the maximal operator $f \mapsto \mathbb{C}_{f}^{*}$ (with $f$ identified with the measure $\left.f \mathscr{H}^{1} \mid \Gamma\right)$ is bounded on $L^{2}(\Gamma)$ (see [CM, D2, $\mathrm{MT}$ ], we have by the Schwarz inequality

$$
\int_{\widetilde{\Gamma}_{m, j}}\left|\int_{\Gamma_{m, j} \backslash B_{z}}(t-z)^{-1} a_{m, j} d t\right| d z \leq c_{8}\left|a_{m, j}\right| \mathscr{H}^{1}\left(\Gamma_{m, j}\right) \leq c_{8} M_{m, j} .
$$

Combining these inequalities with (5), we obtain

$$
\begin{aligned}
& \int_{\{z \in \Gamma: \varepsilon(z)<L 2-m\}}\left|u_{m}\right| d \mathscr{H}^{1} \\
& \leq c_{7} 2^{m} \int_{\Gamma_{\left|j-j_{m}(z)\right| \geq 2 L}} \sum_{m, j}\left(j-j_{m}(z)\right)^{-2} d \mathscr{H}^{1} z \\
&+2^{m} \int_{\Gamma_{\left|j-j_{m}(z)\right|<2 L}} \sum_{m, j} d \mathscr{H}^{1} z+c_{8} 2 L \sum_{j \in \mathbb{Z}} M_{m, j} \\
&= c_{7} 2^{m} \sum_{j \in \mathbb{Z}} M_{m, j} \sum_{|j-k| \geq 2 L}(j-k)^{-2} \mathscr{H}^{1}\left(\Gamma_{m, k}\right) \\
&+2^{m} \sum_{j \in \mathbb{Z}} M_{m, j} \sum_{|j-k|<2 L} \mathscr{H}^{1}\left(\Gamma_{m, k}\right)+c_{8} 2 L \sum_{j \in \mathbb{Z}} M_{m, j} \\
& \leq c_{9}|\mu|\left(R_{m}\right) .
\end{aligned}
$$

Summing over $m$ we get (8).

When $\varepsilon(z) \geq L 2^{-m}$ and $\left|j-j_{m}(z)\right| \geq 2^{m+1} \varepsilon(z)$, we have $R_{m, j} \cap B_{z}=\varnothing$ and $\Gamma_{m, j} \cap B_{z}=\varnothing$. Consequently the same estimates as above yield (9).

To prove (10), first observe that, if $\varepsilon(z) \geq L 2^{-m}$ and $\left|j-j_{m}(z)\right|<2^{m+1} \varepsilon(z)$, then $\Gamma_{m, j} \cup R_{m, j} \subset \widehat{B}_{z}$ with $\widehat{B}_{z}=B(z, 5 L \varepsilon(z))$. Hence,

$$
\begin{aligned}
|w(z)| & \leq \int_{\widehat{B}_{z} \backslash B_{z}}|\zeta-z|^{-1} d|\mu| \zeta+\int_{\Gamma \cap \widehat{B}_{z} \backslash B_{z}}|t-z|^{-1} h(t) d \mathscr{H}^{1} t \\
& \leq \varepsilon(z)^{-1}|\mu|\left(\widehat{B}_{z}\right)+\varepsilon(z)^{-1} \int_{\Gamma \cap \widehat{B}_{z}} h d \mathscr{H}^{1} .
\end{aligned}
$$


The following maximal inequality holds for any positive Borel measure $\nu$ on $\mathbb{C}$; see, for example, [GM]: For all $\lambda>0$,

$$
\mathscr{H}^{1}\left\{z \in \Gamma: \sup _{\varepsilon>0} \varepsilon^{-1} \nu B(z, \varepsilon)>\lambda\right\} \leq c_{10} \lambda^{-1}\|\nu\|_{1} .
$$

Applying this to $|\mu|$ and $h d\left(\mathscr{H}^{1} \mid \Gamma\right)$ and recalling (6), we obtain (10). This completes the proof of the theorem.

\section{EXISTENCE OF PRINCIPAl VAlUes}

We begin by applying Theorem 2.1 on a Lipschitz graph.

3.1. Theorem. If $\mu$ is a finite complex Borel measure and $\Gamma$ a Lipschitz graph on $\mathbb{C}$, then the principal value $C_{\mu}(z)$ exists and is finite for $\mathscr{H}^{1}$ almost all $z \in \Gamma$.

Proof. We may assume $\Gamma$ is compact. We write $\mu=\nu+\sigma$, where $\nu$ is absolutely continuous and $\sigma$ is singular with respect to $\mathscr{H}^{1} \mid \Gamma$. For $\nu$ the principal values exist almost everywhere on $\Gamma$ by [C]. The same follows easily for $\sigma$ once we prove the following statement:

For every $\alpha>0$ there is $\beta>0$ such that there exists $A_{\alpha} \subset \Gamma$ for which $\mathscr{H}^{1}\left(A_{\alpha}\right)<\alpha$ and

$$
\left|C_{\sigma, \delta}(z)-C_{\sigma, \varepsilon}(z)\right| \leq \alpha \quad \text { for } z \in \Gamma \backslash A_{\alpha}, \delta, \varepsilon \in(0, \beta) .
$$

Since $\sigma$ is singular with respect to $\mathscr{H}^{1} \mid \Gamma$, there are for any $\gamma>0$ an open neighborhood $U$ of $\Gamma$ and a compact subset $F$ of $\Gamma$ such that

$$
|\sigma|[(U \backslash \Gamma) \cup(\Gamma \backslash F)]<\gamma \text { and } \mathscr{H}^{1}(F)<\gamma .
$$

We can choose $\beta>0$ such that $\operatorname{dist}(\Gamma, \mathbb{C} \backslash U)>\beta$ and $\mathscr{H}^{1}\left(F_{\beta}\right)<\gamma$, where

$$
F_{\beta}=\{z \in \Gamma: \operatorname{dist}(z, F) \leq \beta\} .
$$

Let $\tau$ be the restriction of $\sigma$ to $(U \backslash \Gamma) \cup(\Gamma \backslash F)$. Then $\|\tau\|_{1}<\gamma$, and

$$
C_{\sigma, \varepsilon}(z)=C_{\tau, \varepsilon}(z) \text { for } z \in \Gamma \backslash F_{\beta}, 0<\varepsilon<\beta .
$$

Let

$$
A=F_{\beta} \cup\left\{z \in \Gamma: C_{\tau}^{*}(z)>\alpha / 2\right\} .
$$

Then, for $\delta, \varepsilon \in(0, \beta)$ and $z \in \Gamma \backslash A$,

$$
\left|C_{\sigma, \delta}(z)-C_{\sigma, \varepsilon}(z)\right| \leq 2 C_{\tau}^{*}(z) \leq \alpha .
$$

By Theorem 2.1,

$$
\mathscr{H}^{1}(A) \leq \gamma+c \alpha^{-1}\|\tau\|_{1}<\left(1+c \alpha^{-1}\right) \gamma .
$$

We obtain the desired inequality by choosing $\gamma$ sufficiently small.

The other results mentioned in the introduction on the existence of principal values almost everywhere on more general rectifiable curves and sets follow from Theorem 3.1 and the fact that any rectifiable curve $\Gamma$ can be written as $\Gamma=A \cup \bigcup_{i=1}^{\infty} \Gamma_{i}$, where $\mathscr{H}^{1}(A)=0$ and each $\Gamma_{i}$ is a Lipschitz graph.

3.2. Remarks. After the first version of this paper was completed, Khavinson showed that the results of $[\mathrm{K}]$ can be used to obtain some related information on 
Cauchy integrals of measures. For example, he proved that if $\Gamma$ is a Lipschitz graph, or more generally a regular arc (see [CM, D2]), and $\mu$ is a finite complex measure on $\mathbb{C}$, then there is a decreasing sequence $\varepsilon_{i} \downarrow 0$ such that

$$
\left|\left\{z \in \Gamma: \sum_{i=1}^{\infty}\left|\hat{\mu}_{i}(z)\right|>\lambda\right\}\right| \leq c \lambda^{-1}\|\mu\|_{1},
$$

where $\mu_{i}$ is the restriction of $\mu$ to $\left\{\zeta: \varepsilon_{i+1}<\operatorname{dist}(\zeta, \Gamma) \leq \varepsilon_{i}\right\}$ and $c$ is an absolute constant. It is not clear whether Khavinson's method can be adapted also to our $\varepsilon$-truncated integrals.

Still later Verdera [V] found a new shorter proof for Theorem 2.1. His methods make use of well-known techniques of harmonic analysis.

\section{REFERENCES}

[C] A. P. Calderón, Commutators, singular integrals on Lipschitz curves and applications, Proc. Internat. Congr. Math. (Helsinki, 1978), Acad. Sci. Fenn., Helsinki, 1980, pp. 85-96.

[CM] M. Christ, Lectures on singular integral operators, CBMS Regional Conf. Ser. in Math., vol. 77, Amer. Math. Soc., Providence, RI, 1990.

[D1] G. David, Opérateurs integraux singuliers sur certaines courbes du plan complexe, Ann. Sci. École Norm. Sup. (4) 17 (1984), 157-189.

[D2] _ Wavelets, Calderón-Zygmund operators, and singular integrals on curves and surfaces, Lecture Notes in Math., vol. 1465, Springer-Verlag, New York, 1991.

[F] K. J. Falconer, Geometry of fractal sets, Cambridge Univ. Press, Cambridge, 1985.

[GT] T. W. Gamelin, Uniform algebras, Chelsea, New York, 1984.

[GJ] J. B. Garnett, Analytic capacity and measure, Lecture Notes in Math., vol. 297, SpringerVerlag, New York, 1972.

[GM] M. de Guzman, Differentiation of integrals in $\mathbb{R}^{n}$, Lecture Notes in Math., vol. 481, Springer-Verlag, New York, 1975.

[K] D. Khavinson, F. and M. Riesz theorem, analytic balayage, and problems in rational approximation, Constr. Approx. 4 (1988), 341-350.

[M] P. Mattila, Cauchy singular integrals and rectifiability of measures in the plane, Adv. in Math. (to appear).

[MT] T. Murai, A real variable method for the Cauchy transform and analytic capacity, Lecture Notes in Math., vol. 1307, Springer-Verlag, New York, 1988.

[V] J. Verdera, A weak type inequality for Cauchy transforms of measures, Publ. Mat. 36 (1992), 1029-1034.

Department of Mathematics, University of Jyväskylä, P.O. Box 35, SF-40351 JYVÄSKYLÄ, FINLAND

Department of Mathematics, Moscow State University, 117234 Moscow, Republic of RUSSIA

Current address: Department of Mathematics, University Autonoma de Barcelona, Campus Universitario, 08193 Barcelona, Spain 